\title{
INDIRECT-DIRECT HYBRID TARGET EXPERIMENTS WITH GEKKO XII LASER
}

\author{
H. NISHIMURA, H. AZECHI, K. FUJITA, M. HEYA, R. ISHIZAKI*, N. IZUMI, \\ N. MIYANAGA, M. MURAKAMI, M. NAKAI, S. NAKAI, M. NAKATSUKA, \\ M. NISHIKINO, K. NISHIHARA, T. NORIMATSU, Y. OCHI, N. OHNISHI, \\ K. SHIGEMORI, H. SHIRAGA, H. TAKABE, T. YAMANAKA, C.YAMANAKA**, \\ and K. MIMA \\ Institute of Laser Engineering, Osaka University, \\ 2-6 Yamada-oka, Suita, Osaka 565-0871, Japan
}

\begin{abstract}
A new fusion-capsule drive-scheme was investigated. The capsule is illuminated by an low-intensity thermal $\mathrm{x}$-ray pulse in prior to the main drive pulse, which leads to noticeable suppression of initial imprinting, brought by the drive beam, due to thermal smoothing in the preformed plasma. Among several types of the indirect-direct hybrid targets, we investigated the indirect-direct hybrid effect for two types: One is a foamhybrid in which the capsule has a low-density foam layer attached directly on its surface and pulsed radiation generated with a thin high $\mathrm{Z}$ layer on the foam propagates through the foam, creating a preformed plasma. The other is an external-hybrid in which the capsule is illuminated, apart from the capsule, by external x-ray radiation generated with different beams from the capsule drive beams. The hybrid effect was demonstrated by imposing initial imprint on a planar target with an intensity modulated beam and subsequent nonuniformity growth due to Rayleigh-Taylor instability was observed by face-on back-lighting. The observed suppression due to presence of the preformed plasma is interpreted by the cloudy-day model for both hybrids. Capsule implosion experiments have been also started. Overall implosion dynamics observed is replicated by 1D hydrocode simulations. Preliminary results form the implosion experiments are presented.
\end{abstract}

\section{INTRODUCTION}

As a mile-stone of inertial confinement fusion (ICF) research, it is of great importance to create a hydrodynamically equivalent plasma (HEP), similar to that of central-spark ignition but in a smaller scale, with precisely controlled multiple laser beams. For such an isobaric compression scheme, hydrodynamic instabilities arising from surface roughness of the fuel capsule and nonuniformity of laser irradiation must be suppressed below a tolerable level to attain high fusion gain.

At the Institute of Laser Engineering (ILE), Osaka University, the beam profile and the power balance among the 12 beams of GEKKO XII Nd: glass laser have been extensively improved [1] in order to carry out the precise implosion experiments. Figure 1 illustrates the present status and approach to high gain. Initial perturbation of amplitude $A_{0}$ on a capsule surface may grow due to Rayleigh-Taylor

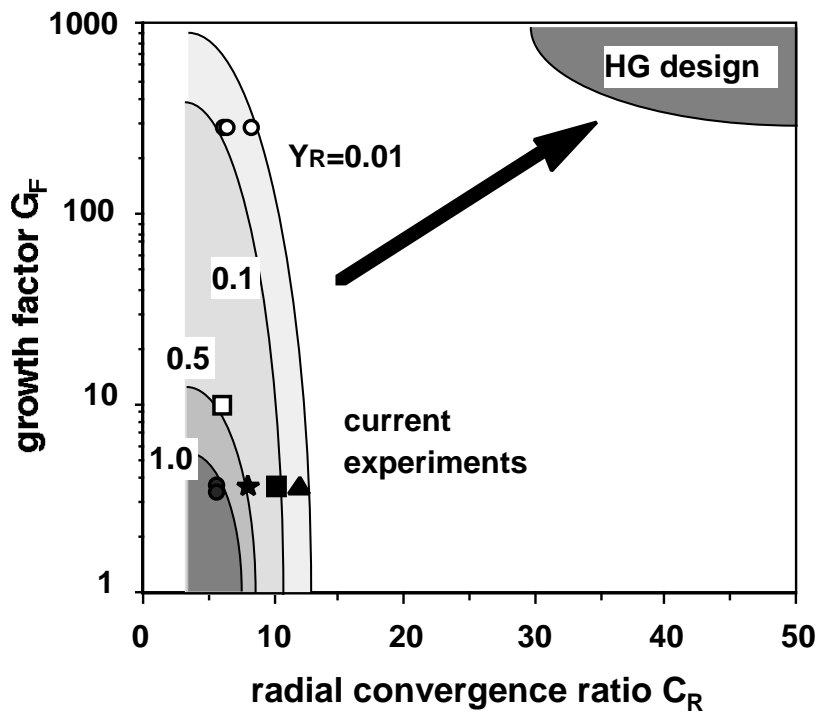

FIG. 1 Present status and future goal of implosion experiments. Circles represent the experimental data for low-isentrope (open circles) and mediumisentrope (closed circles) cases [2].

\footnotetext{
* National Institute for Fusion Science, 322-6 Shimoishi-cho, Toki, Gifu 509-5292, Japan

** Institute for Laser Technology, 1-8-4 Utsubohonmachi, Nishi-ku, Osaka 550-0004, Japan
} 
(R-T) instability during the acceleration phase. The perturbation fed through the inner surface of the capsule together with the initial inner-surface roughness will grow subsequently in the deceleration phase. The overall perturbation amplitude on the capsule-fuel interface (given by $G_{F} A_{0}$ using a growth factor $G_{F}$ ) must be smaller than the final radius of the compressed fuel $R_{f}$. This condition is given as $G_{F} C_{R}<f\left(R_{\delta} / A_{0}\right)$ where the radial convergence ratio of $C_{R}$ is defined by $R_{d} / R_{f}\left(R_{o}\right.$ is the initial gas-fuel radius) and $f$ is a fractional depth of the mixing region normalized with the final radius $R_{f}$ The criteria for different $\mathrm{G}_{F}$ and $C_{R}$ are represented by curves with yield ratios $Y_{R}\left(=Y_{\text {exp }} / Y_{I D \text {-sim }}\right)$. A picket-pulse followed by a square-drive-pulse was used to control the instability growth during the acceleration phase at the expense of larger isentrope [2]. Figure 1 summarizes the results of the implosion experiments using plastic capsules (as a surrogate of main fuel) containing $\mathrm{D}_{2}$ fuel (as a hot spark). As represented by closed circles (for the time gap between the two drive pulses of $400 \mathrm{ps}$ ), the observed neutron yields agree well with those from 1D simulation under relatively low convergence of 6 , growth factor of 4, and medium adiabat of 4-5. This is in contrast to low-adiabat but much greater R-T instability with a picket-pulse-free, single square drive (shown with open circles). Hydrocode simulations showed, with the picket pulse, larger reduction of R-T growth rates from the medium to higher mode $(l=30-500)$ but not in the low modal numbers [2]. Other closed symbols represent the results for lower fuel pressures, resulting in larger radial convergence and larger deviation from 1D simulations. Thus, even with the picket pulse, fusion output is also sensitive on the radial convergence, which may be attributed to the remaining low modal nonuniformities brought by twelvebeam irradiation.

In order to achieve greater $C_{R}$ with lower adiabat (which results in greater $G_{F}$ ), smoothing by spectral dispersion (SSD) is to be implemented to the laser system in addition to the partially coherent light (PCL) [1]. The PCL will pre-compress the capsule uniformly as a foot-pulse, and the 2 dimensional (2D) SSD light will accelerate it as a main drive, providing a lower adiabat compression. Initial imprint, however, still remains as an obstacle for high convergence since PCL takes several tens ps to manifest its excellent effect. To eliminate high modal nonuniformities arising from the initial imprint, indirect-direct hybrid scheme [3] will be used in a concept shown in Fig. 2. This is a combination of a uniform indirect-drive in the start-up phase and efficient direct-drive afterwards. The idea is that the capsule surface is irradiated with low-intensity thermal $\mathrm{x}$-ray radiation to generate uniform plasma expansion without imprint in prior to the main drive incidence. At the time when the main drive starts, the capsule already wears a buffer layer consisting of the expanding low density plasma. Thus initial imprinting imposed by the main drive will be mitigated substantially by thermal

(a) direct-drive

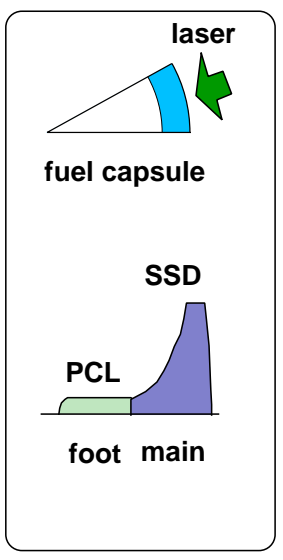

(b) indirect-direct hybrid drive

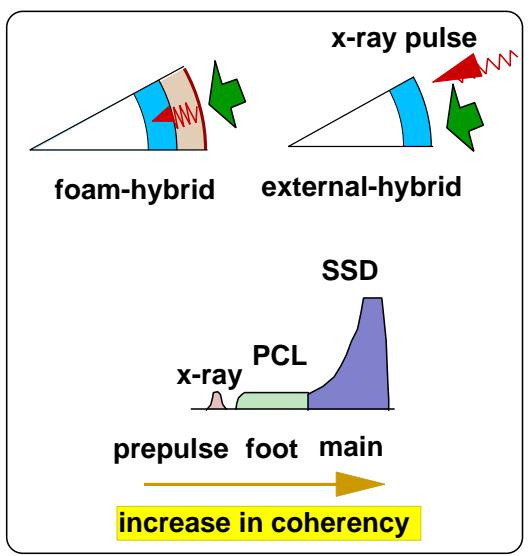

(c) indirect-drive

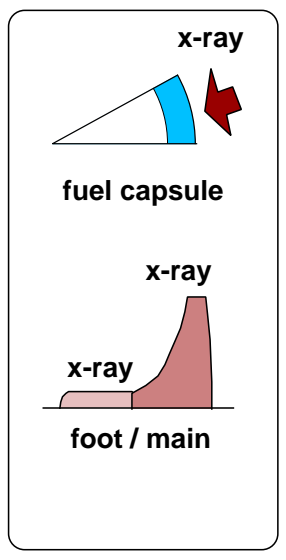

FIG. 2 Concept of indirect-direct hybrid drive. An x-ray prepulse irradiates first the capsule surface to generate a preformed plasma, which mitigates initial imprint caused by the PCL foot and SSD main drive. Thus coherency of the pulses increases sequentially. For comparison, nominal direct-drive and indirect-drive schemes are shown respectively in (a) and (c). 
smoothing taking place between the energy deposition region and the ablation region. Their separation is called stand-off distance $(D s)$. The other important effect is that the preformed surface plasma is optically opaque so that it can be a protection from disintegration of the capsule in an initial stage of heating caused by laser light shine-through [4].

Among the several hybrid-targets, we examined the hybrid effects for two types: One is called foam-hybrid in which the capsule has a low-density foam layer directly attached on the surface of the capsule [5] and $\mathrm{x}$-ray radiation generated with a thin high $\mathrm{Z}$ layer set on the foam surface propagates supersonically through the foam, creating a preformed plasma [6]. The other is called external-hybrid in which the capsule is illuminated by an x-ray flash generated externally with a different beam from the main drive [3].

A series of experiments were carried out using planar targets to quantify the smoothing effects of the hybrid targets by imposing initial imprints with a spatially modulated laser beam from GEKKO XII. The smoothing effect by the buffer layer or the preformed plasma was investigated by observing growth of fluid instability in linear regime of R-T instability by varying target and $\mathrm{x}$-ray flash condition. Of particular interest are to find an optimum condition to avoid excessive radiative heating which may cause capsule disassembly resulting in degradation of implosion performance, and to investigate influence of long wavelength perturbation on convergence uniformity which may not be excluded even with the preformed plasma. Time evolution of areal density fluctuations $(\Delta \rho r)$ due to the instability growth were observed by means of face-on x-ray backlighting.

As a consequence of these planar experiments, target implosion experiments with $\mathrm{D}_{2}$ filled spherical capsules were performed for both type hybrids as an integrated spherical experiment.

\section{IMPRINT MITIGATION EXPERIMENTS WITH PLANAR TARGETS}

\subsection{Foam-hybrid targets}

Frequency doubled PCL pulses from GEKKO XII were used in the experiment. Initial imprint on a planar target surface was given with the intensity modulated beam. Wavelength of the modulation $(\lambda p)$ was either 40 or $88 \mu \mathrm{m}$ and depth was $20 \%$ of average intensity. Waveform of the imprint beams was a square of $50 \mathrm{ps}$ rise and $2.2 \mathrm{~ns}$ duration in FWHM. Average irradiance of the imprint beam was $4 \times 10^{12} \mathrm{~W} / \mathrm{cm}^{2}$. The main drive started $1 \mathrm{~ns}$ later than the imprint beam for the $88 \mu \mathrm{m}$ and $0.6 \mathrm{~ns}$ later for the $40 \mu \mathrm{m}$. In the latter case the shock wave generated by the main drive temporally coincides with that generated by the imprint beam at the target rear side. This was confirmed by observing emanation of visible light due to shock arrival at the target rear-side with an optical streak camera. Waveform of the main drive was same as the imprint beam. Irradiance of the main drive was $7 \times 10^{13} \mathrm{~W} / \mathrm{cm}^{2}$.

Targets used consisted of $20 \mu \mathrm{m}$-thick plastic foam $\mathrm{C}_{24} \mathrm{H}_{18} \mathrm{O}_{4}$, formed onto $1.11 \mathrm{~g} / \mathrm{cc}$-density and $8 \mu \mathrm{m}$-thick parylene $\mathrm{C}_{8} \mathrm{H}_{8}$ foils. Density of the foam was $80 \mathrm{mg} / \mathrm{cc}$ for the $88 \mu \mathrm{m}$ and $40 \mathrm{mg} / \mathrm{cc}$ for the $40 \mu \mathrm{m}$ cases. Foam surface for the $88 \mu \mathrm{m}$ was overcoated directly with $20 \mathrm{~nm}$-thick gold, however, that for the $40 \mu \mathrm{m}$ was first covered with a parylene foil of $0.15 \mu \mathrm{m}$-thick then 5 , or $10 \mathrm{~nm}$ thick gold were overcoated for each target (see Fig. 3). This treatment is important to avoid diffusion of the gold coat deep into the foam porous structure, thus to improve controllability of the x-ray flash generation and simplify hydrocode modeling. Single plastic foils were also used as a baseline. Foils of $\mathrm{C}_{6} \mathrm{H}_{7} \mathrm{~F}_{3} \mathrm{O}_{2} 9.1 \mu \mathrm{m}$-thick and $1.18 \mathrm{~g} / \mathrm{cc}$-density were used for the $88 \mu \mathrm{m}$ and parylene foils of $10.6 \mu \mathrm{m}-$ thick were for the $40 \mu \mathrm{m}$. Note that the areal densities for these targets were chosen to be almost same as those of the foam buffered targets.

Time resolved $\mathrm{x}$-ray shadowgraphs were taken with an $\mathrm{x}$-ray streak camera coupled to an imaging slit of $10 \mu \mathrm{m}$-width. Spatial resolution and resultant modulation transfer function were measured separately by taking a streaked shadow image of a Fresnel zone plate made of gold. Temporal resolution of the system was 90 ps. X-ray backlighting source was L-line emissions (1.1-1.3 $\mathrm{keV}$ ) from laser generated copper plasma. Figure 3 shows $\mathrm{x}$-ray backlight streak images for different type of foam hybrid targets. The modulation wavelength is $40 \mu \mathrm{m}$. The gross areal density perturbation was first derived from opacity fluctuations observed in the face-on backlight image then decomposed into individual sinusoidal perturbation components by taking the modulation transfer 
function into account.

Figure 4 shows the perturbation growth for the $88 \mu \mathrm{m}$ wavelength. The areal density perturbation for the $\mathrm{C}_{6} \mathrm{H}_{7} \mathrm{~F}_{3} \mathrm{O}_{2}$ foil grows almost linearly up to $1.3 \mathrm{~ns}$. Perturbation growth for the foam-buffered target shows a similar trend. But a factor of 2 lower amplitudes than that of the single foil are obtained in the linear growth regime. In addition a large reduction of the perturbation amplitude is seen after $1.2 \mathrm{~ns}$. This reduction disappears when thinner gold overcoating on the thin parylene were adopted as shown in Fig. 5. Although the no-coat and $5 \mathrm{~nm}$ coat targets show no remarkable difference, the root-meansquare (rms) amplitudes over 13-512 $\mu \mathrm{m}$-wavelength become smaller for thicker coat targets among these targets. On the contrary, as seen in Fig. 3, thicker coat targets tend to pick-up a very long wavelength nonuniformity included in the imprint and/or drive beams. This trend was more obvious for the $88 \mu \mathrm{m}$ case. From the hydrocode simulations and mode predictions, this is attributed to a larger pressure-perturbation due to better light absorption for thicker coating and subsequent growth of the perturbation caused by a ripple shock wave propagating through the foam and the foam/plastic interface [7]. For the present conditions, gold overcoat of $5 \mathrm{~nm}$ was chosen as an optimum $\mathrm{x}$-ray converter.

Suppose that the thermal smoothing effect via heat conduction region is expressed with a reduction factor of $\exp (-k D s)$ where $k$ is the wavenumber of the perturbation, one may simply obtain the effective stand-off distance from the experimental results above. By using the reduction factors of 2 for the $88 \mu \mathrm{m}$ perturbation ( $20 \mathrm{~nm}$ gold coating) and 5.5 for the $40 \mu \mathrm{m}$ perturbation $(10 \mathrm{~nm}$ gold coating), $D s^{\prime}$ for both cases become uniquely about $11 \mu \mathrm{m}$. Simulation of the foam-target acceleration was made with one dimensional code ILESTA-1D [8]. Typical result is shown in Fig. 6. The distance between the critical point (represented by $n_{c}$ ) and the ablation front defined as the pressure maximum point $\left(P_{\max }\right)$ becomes longer monotonically after the onset of the imprint pulse. $D s$ becomes around 12 $\mu \mathrm{m}$ at the onset of the drive beam, which is in good agreement with the experiments.

\subsection{External-hybrid targets}

Similar to the case of the foam-hybrid targets, imprint mitigation was investigated for the

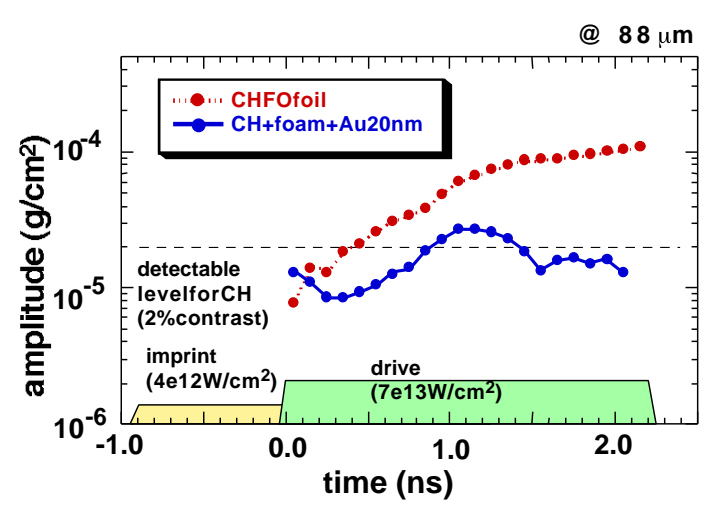

FIG. 4 Time evolution of the imposed perturbation for the plane foil and foam-buffered target. Perturbation wavelength is $88 \mu \mathrm{m}$.

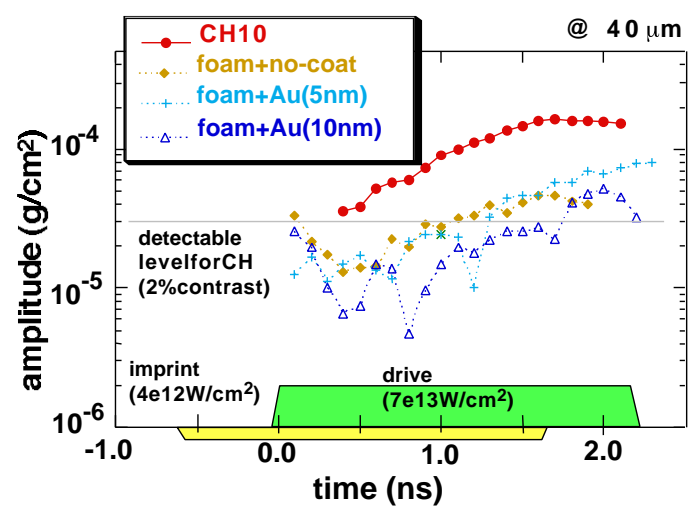

FIG. 5 Growth of initial imprinting for the simple foil and foam-hybrid targets. Perturbation wavelength is $40 \mu \mathrm{m}$. 


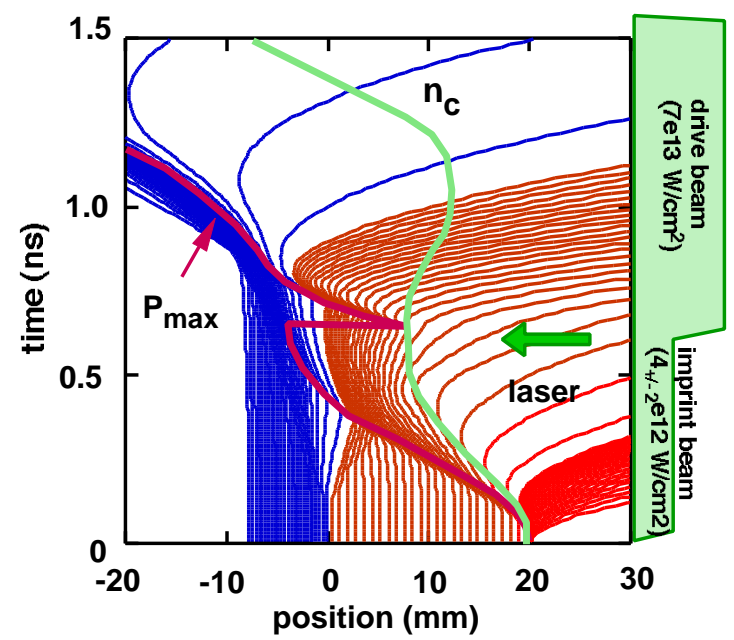

FIG. 6 Flow diagram of the form hybrid target simulated with ILESTA-1D.

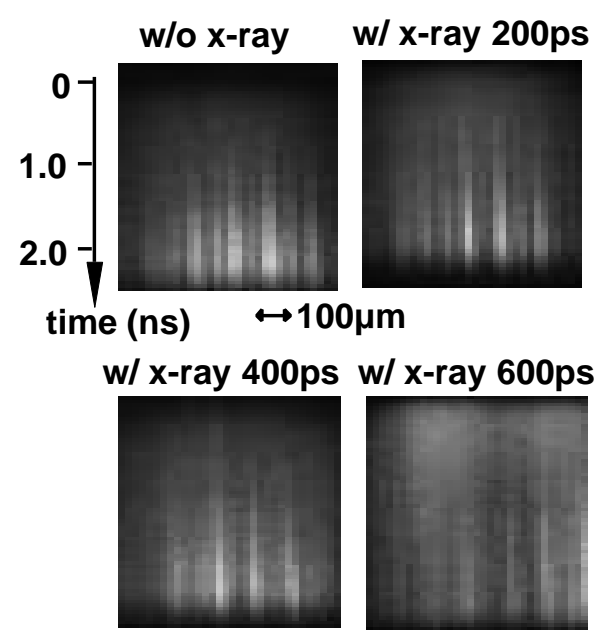

FIG. 7 X-ray backlight streak images of with and without $x$-ray pre-irradiation.

external-hybrid. Surface perturbation was seeded by the intensity modulated PCL beam with wavelengths of $40 \mu \mathrm{m}$ to $120 \mu \mathrm{m}$ and rms depth of $40 \%$ of average (a $1.0 \mathrm{~ns}$ flat-top pulse of $5 \times 10^{12} \mathrm{~W} / \mathrm{cm}^{2}$ intensity). A gold plate was set $1.8 \mathrm{~mm}$ away from the planar targets as an external x-ray source for pre-plasma formation. The gold plate was irradiated with a separate beam at an intensity of $5 \times 10^{13}$ $\mathrm{W} / \mathrm{cm}^{2}$ of a flat top shape which started $0.2,0.4$ or $0.6 \mathrm{~ns}$ prior to the imprint pulse, providing $\mathrm{x}$-ray irradiance of $7 \times 10^{10} \mathrm{~W} / \mathrm{cm}^{2}$ on the foil. Thickness of the foil was $16 \mu \mathrm{m}$.

The imposed surface perturbation was amplified via Rayleigh-Taylor instability during the main drive irradiation (a $2.2 \mathrm{~ns}$ flat-top pulse of $1 \times 10^{14} \mathrm{~W} / \mathrm{cm}^{2}$ intensity). Resulting areal density perturbations were observed with the same x-ray probe and the streak camera as in the foam-hybrid case. Experiments were performed with single- and multi-mode perturbations. Typical streak images for the multi-mode experiments are shown in Fig. 7. Similar to those obtained previously for singlemode perturbations [3], the imprint structure is significantly reduced with increase in the x-ray preirradiation time for the imposed perturbations. In contrast to the foam-hybrid case, no remarkable growth of long wavelength perturbation is seen.

Growth of perturbation of $\lambda p=40 \mu \mathrm{m}$ with and without $\mathrm{x}$-ray pre-irradiation is plotted in Fig.8. $\mathrm{R}-\mathrm{T}$ growth in the linear phase is seen in the middle way for each case. It is shown clearly that the

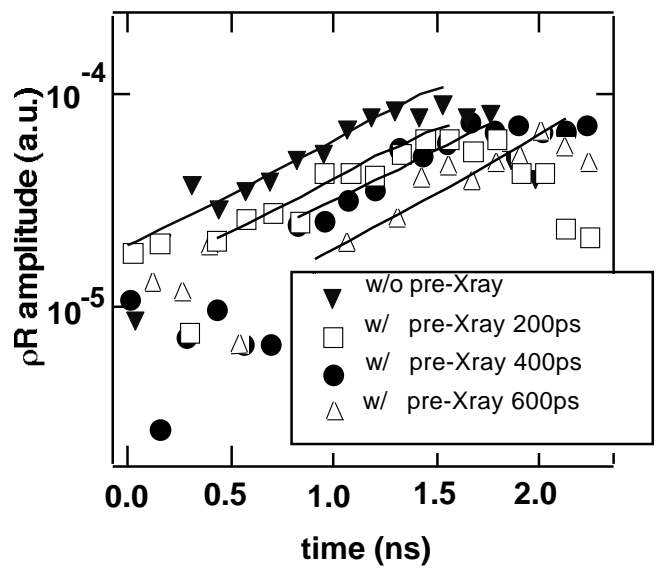

FIG. 8 Growth of the perturbation amplitudes for cases with and without $x$-ray preirradiation.

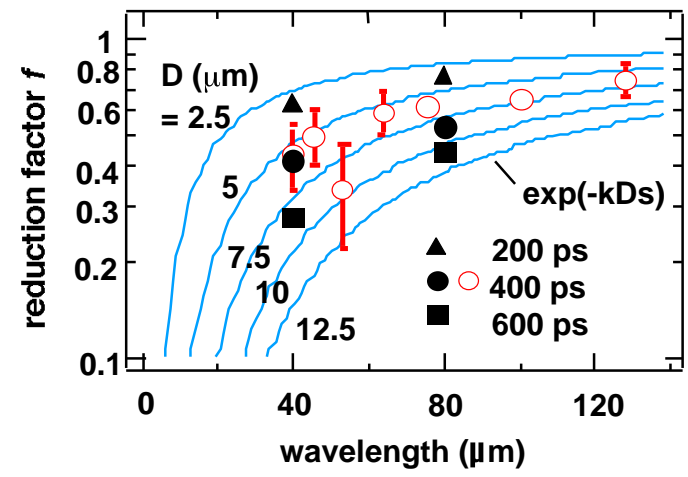

FIG. 9 Reduction of perturbation growth for various perturbations and pre-irradiation times is compared with a cloudy day model. 
amplitude for the x-ray pre-irradiation is mitigated by a factor of 1.4-2.5 depending on the preirradiation time. Observed smoothing effect can also be explained by the simple cloudy-day model. Figure 9 shows a comparison of experimental results with the model for $\lambda p=40,46,53,64,80$ and $120 \mu \mathrm{m}$. Reduction of perturbation was more effective for shorter wavelength and longer preirradiation time. The data points are consistent with $D s=5-7.5 \mu \mathrm{m}$ for x-ray pre-irradiation time of 0.4 ns, which is replicated well by ILESTA-1D.

\section{IMPLOSION OF SPHERICAL TARGETS}

\subsection{Foam-hybrid targets}

A monolithic type of foam-hybrid fusion capsule was newly developed for use in the implosion experiments. Polymalization of the foam layer as well as the plastic shell (as a surrogate of dense fuel) was made under a sequence of fabrication by the emulsion method [9]. This ensures uniform layer formation and intimate contact between the foam layer and the shell. Figure 10 shows its schematic structure (a), optical image (b), and x-ray radiograph (c). The intensity profile of the x-ray radiograph was used to measure density, thickness, and uniformity of the layers. To seal a deuterium and argon mixture gas in the capsules, they were kept at $80 \mathrm{~K}$ with a cooling system just before the laser shoot. The fill pressure measured at room temperature was $\mathrm{D}_{2}: \mathrm{Ar}=30: 0.075 \mathrm{~atm}$. The high fill pressure was chosen to ensure the low radial convergence, thus high-light the smoothing effect for high modal nonuniformity.

The laser used was twelve beam, green PCL pulses from GEKKO XII. Energy was totally 1.8 $\mathrm{kJ}$ on target and waveform of the beams was a square of $50 \mathrm{ps}$ rise and $2.2 \mathrm{~ns}$ duration, yielding irradiance of $1.1 \times 10^{14} \mathrm{~W} / \mathrm{cm}^{2}$ on the target surface.

Implosion dynamics and hot core formation were observed with two $\mathrm{x}$-ray framing cameras (XFC) and a novel $\mathrm{x}$-ray monochromatic framing camera (MXFC). Temporal resolution of XFC was 80 ps with a 100 ps frame-interval. MXFC provided monochromatic images of Ar Ly $\beta$ and He $\beta$ lines from the hot core. Time resolution was 40 ps with a 50 ps frame-interval. Implosion performance were measured with x-ray spectrometers with and without temporal resolutions, neutron detectors, and a multi-channel neutron spectrometer.

Figure 11 shows a representative $\mathrm{x}$-ray frame images for the foam-hybrid targets. The observed implosion dynamics was compared with 1D hydrocode simulations which were post processed with an $\mathrm{x}$-ray emission image synthesizer. A good agreement was obtained in the comparison, showing that overall understanding of energy deposition and transport is obtained. However, as seen in the images in Fig. 11, low-mode nonuniform emission appears in later time. The neutron yield ratio $Y_{R}$ was about a factor of 4 lower than that for the conventional single plastic shell implosion. In conjunction to the growth of low-mode nonuniformity found in the planar target experiments, investigations on the degradation of fusion performance and perturbation growth through the foam layer and foam/shell interface are underway by the analytical model and multi-dimensional hydrocode simulations including non-local heat transport treatment (necessary for thermal smoothing) and non-LTE multi-group radiation transport (for radiation smoothing).

\subsection{External-hybrid targets}

Implosion experiments of the indirect-direct hybrid-drive spherical capsules with external $x$-ray sources have also been started. Figure 12 shows a schematic of the target-beam configuration. The target consisted of an inner

C

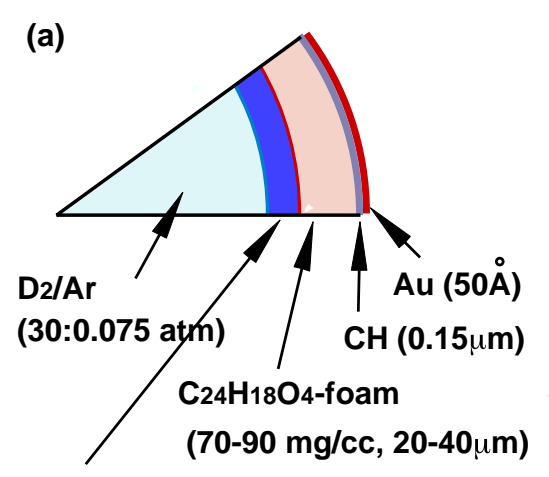

$\mathrm{C}_{38} \mathrm{H}_{66} \mathrm{O} 12$ (5-7 $\left.\mu \mathrm{m}\right)$
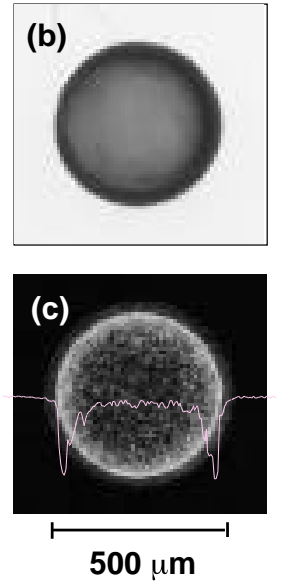

FIG. 10 Foam-hybrid targets for implosion experiments. 


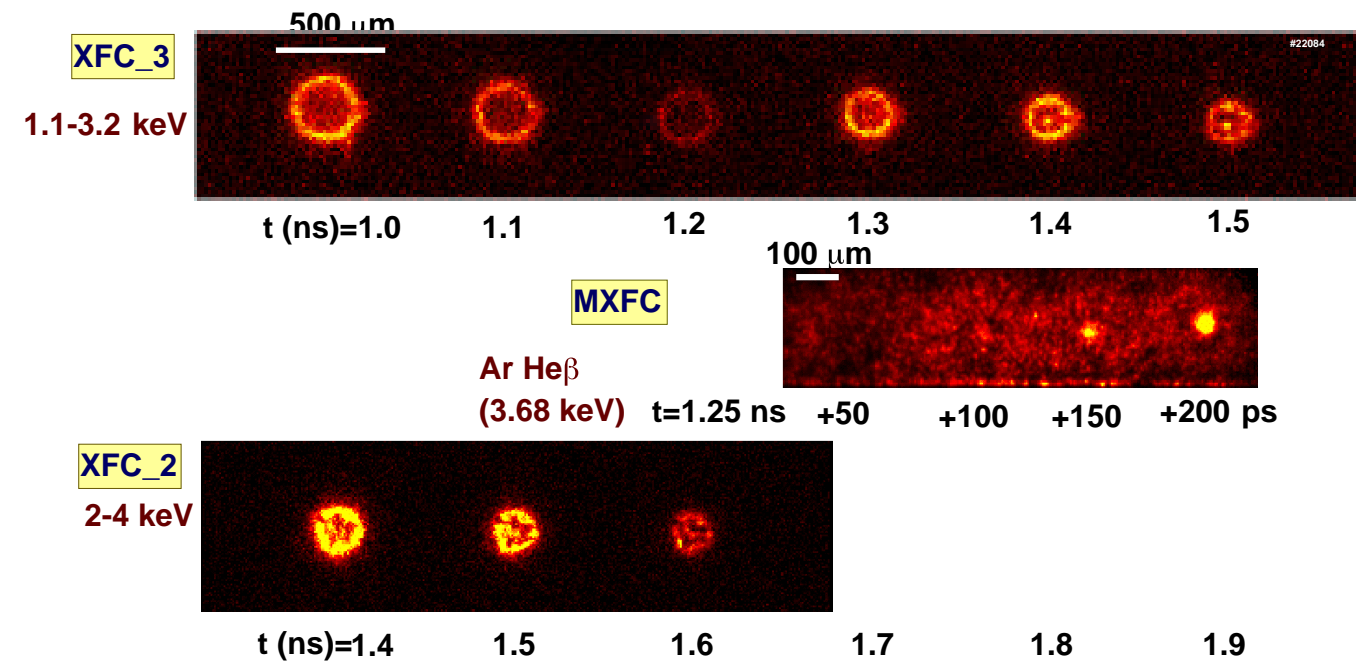

FIG. $11 X$-ray frame images of foam-hybrid targets. Time was measured from the onset of the drive pulse.

capsule ( $\mathrm{CH}$ shell containing a $\mathrm{D}_{2} / \mathrm{Ar}$ mixture gas) and an outer shell made of gold as an external x-ray emitter. The diameters and wall thicknesses of the capsule and the gold shell were respectively 480 and 7.8, and 3000 and $15 \mu \mathrm{m}$. The inner capsule was mounted with a straight carbon fiber of $6 \mu \mathrm{m}$ diameter onto the inner surface of the gold shell. The assembled targets were also cooled down to 80 $\mathrm{K}$ to fill the fuel gas of $30 \mathrm{~atm}$ measure at room temperature.

The target was irradiated with green beams from GEKKO-XII. Central part ( $1 / 3$ of full diameter) of each laser beam was bent by a pair of wedge plates to point the inner surface of the gold shell at the position allotted as a respective external x-ray source, while the remaining annular beam irradiated the capsule as a main drive. (Irradiation uniformity was maintained by the use of random phase plates). The central and the annular beams were injected through inlet slits (600 $\mu$ m-wide) and inlet holes $(1200 \mu \mathrm{m}$-diam) made on the gold shell. Twelve beams for main drive irradiated the capsule in a dodecahedral symmetry, while the twelve x-ray sources were in a nearly dodecahedral symmetry. The main drive beams were PCL with an energy of $200 \mathrm{~J} / \mathrm{beam}$ in a $2.0 \mathrm{~ns}$ flat top, while the central beams were coherent laser light with an energy of $5 \mathrm{~J} /$ beam in a $0.1 \mathrm{~ns}$ Gaussian pulse which started $0.4 \mathrm{~ns}$ prier to the main drive.

Implosion was performed successfully. Figure 13 shows a series of x-ray framing pictures, which indicate that the capsule imploded nearly in a spherical symmetry. However, its behavior can be reproduced in the simulation assuming $75+/-5$ $\%$ of laser energy is deposited on the fuel capsule. This may suggest occurrence of voidclosure between the capsule and gold shell with expanding plasmas or laser light refraction by the plasma from $\mathrm{x}$-ray sources. On this assumption, the yield ratio $Y_{R}$ was very close to that for the standard implosion of a plastic shell without external $\mathrm{x}$-ray irradiations. We have no definite explanation for this issue at this moment and need further investigation.

\section{CONCLUSION}

A new scheme of indirect-direct hybrid targets has been investigated as a key capsule design for advanced implosion performances.

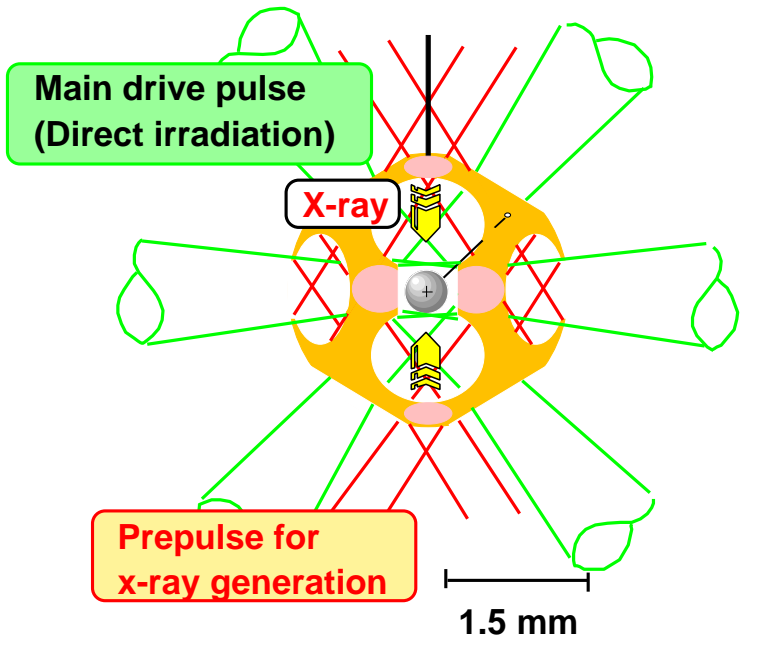

FIG. 12 Schematic of the beam-target configuration of an indirect/direct hybrid drive implosion. 


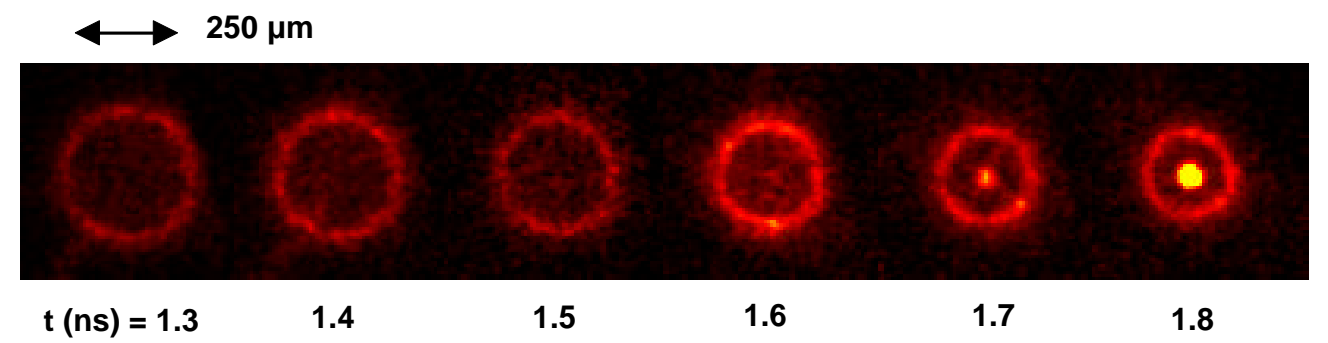

FIG. 13 Sequential $x$-ray framing pictures of an external-hybrid target implosion .

The hybrid effect was demonstrated by imposing initial imprint on a planar target with an intensity modulated beam. The observed suppression is well interpreted by the cloudy-day model for both hybrid targets. Capsule implosion experiments have been also started. Overall implosion dynamics observed is replicated by 1D hydrocode simulations. However, the hybrid effect has not clearly revealed in the fusion performances. This might be partly due to inherent dominance of low-modal nonuniformity. Further investigation will be made, including improvement in the capsule deign and fabrication technology, in order to demonstrate the feasibility of the hybrid targets for high fusion gain.

\section{Acknowledgements}

The authors sincerely acknowledge E. Foerster, I.Uschmann, and R. Butzbach for their invaluable contributions to the development of monochromatic framing camera used for the first time in this study. They also thank experimental crew of the Gekko XII facility. All their efforts are gratefully appreciated. This work was supported in part by Japan-Germany Scientific Collaboration Program of Japan Society for the Promotion of Science.

\section{REFERENCES}

[1] MIYANAGA, N., et al., Improvement of laser irradiation nonuniformity in Gekko XII laser system, SPIE Proc. 2633 (1995) 183- and references therein.

[2] MIMA, K., et al., Recent progress of implosion experiments with uniformity-improved GEKKO XII laser facility at the Institute of Laser Engineering, Osaka University, Phys. Plasmas 74 (1996) 2077-2083.

[3] SHIRAGA, H., et al., Experiments on indirect/direct-hybrid drive scheme, 16th IAEA Fusion Energy Conference, Montreal, Canada, 7-11 Oct. 1996, IAEA-CN-64/B2-4.

[4] BRADLEY, D.K., et al., Early-time "shine-through" in laser irradiated targets, Laser Interaction and Related Plasma Phenomena, edited. by H. Hora and G. Miley (Plenum Press. N.Y. 1991), 9, 323-334.

[5] OKADA, K., et al., Laser implosion of thick low-Z foam coated glass micro balloon, Apply. Phys. Lett. 42 (1983) 231-233,

[6] DESSELBERGER, M., et al., Use of x-ray preheated foam layer to reduce beam structure imprint in laser -driven targets, Phys. Rev. Lett., 74 (1995) 2961-2964.

[7] ISHIZAKI, R. et al., Instability of a contact surface driven by a nonuniform shock wave, Phys. Rev. E 53 (1996) R5592-R5595.

[8] TAKABE, H., et al., Scaling of implosion experiments for high neutron yield, Phys. Fluids 10 (1988), 2884-2893.

[9] TAKAGI, M., et al., Development of foam shell with plastic ablator for cryogenic laser fusion target, J. Vac. Sci. Technol., A11 (1993) 2837-2845. 